\title{
Results of the first 1,000 procedures after resumption of elective orthopedic services following COVID-19 pandemic: Experiences of a high-volume arthroplasty center
}

\author{
Thorsten Gehrke, $\mathrm{MD}^{1 *} \mathbb{D}$, Philip Linke, $\mathrm{MD}^{1 *} \mathbb{D}$, Amir Sandiford, $\mathrm{MD}^{2} \mathbb{1}$, Christian Lausmann, $\mathrm{MD}^{1}$ (D), \\ Mustafa Citak, MD, $\mathrm{PhD}^{1}$ (1D \\ 'Department of Orthopaedic Surgery, ENDO-Klinik Hamburg, Holstenstr, Hamburg, Germany \\ 2Joint Reconstruction Unit, Southland Hospital, Kew Road, Kew, Intercargill, New Zealand
}

The pandemic of severe acute respiratory syndrome-coronavirus 2 (SARS-CoV-2), also named as novel coronavirus-2019 (COVID-19), has rapidly become one of the most significant socioeconomic and medical events in the modern human history. ${ }^{[1,2]}$ It is widely acknowledged that lockdown, social distancing, and attention to hand hygiene have led to a reduction in the number of cases. ${ }^{[3-5]}$ However, despite these measures, healthcare institutions across the Europe and North America were at risk of being overwhelmed by the large numbers of patients requiring respiratory support, as well as the need for isolation of the infected patients. ${ }^{[6]}$

Received: November 24, 2020

Accepted: December 01, 2020

Published online: January 06, 2021

Correspondence: Mustafa Citak, MD. Department of

Orthopaedic Surgery, Helios ENDO-Klinik Hamburg

Holstenstrasse 2, 22767 Hamburg, Germany.

E-mail: mustafa.citak@helios-gesundheit.de

Doi: 10.5606/ehc.2021.80198

* Thorsten Gehrke and Philip Linke contributed equally to the writing of this manuscript.

Citation: Gehrke T, Linke P, Sandiford A, Lausmann C, Citak M. Results of the first 1,000 procedures after resumption of elective orthopedic services following COVID-19 pandemic: Experiences of a high-volume arthroplasty center. Jt Dis Relat Surg 2021;32(1):3-9.

C2021 All right reserved by the Turkish Joint Diseases Foundation

This is an open access article under the terms of the Creative Commons Attribution-NonCommercial License, which permits use, distribution and reproduction in any medium, provided the original work is properly cited and is not used for commercial purposes (http://creativecommons.org/licenses/by-nc/4.0/).

\section{ABSTRACT}

Objectives: This study aims to investigate the effectiveness of a screening questionnaire to identify high-risk patients for novel coronavirus-2019 (COVID-19) among those undergoing elective orthopedic surgery.

Patients and methods: Between May $4^{\text {th }}, 2020$ and June $11^{\text {th }}$, 2020, a total of 1,021 consecutive patients (492 males, 529 females; mean age: $62.3 \pm 15.1$ years; range, 13 to 91 years) who were scheduled for elective orthopedic surgery were included. A screening questionnaire was applied to all patients. The patients admitted to hospital were also tested for COVID-19 infection through reverse transcription-polymerase chain reaction of the nasopharyngeal swab.

Results: Of the patients, 1,003 (98.2\%) underwent elective surgery as planned. The screening questionnaire classified 30 patients as high-risk for COVID-19. A total of 18 procedures $(n=18,1.8 \%)$ were postponed due to the high risk of possible transmission of COVID-19. None of 991 low-risk patients were tested positive for COVID-19.

Conclusion: The use of guiding principles for resuming elective orthopedic surgery is safe without a higher risk for complications in selected cases.

Keywords: COVID-19, elective surgery, orthopedic surgery, screening.

One of the strategies to mitigate the spread of COVID-19 was the halting of elective surgical services in an attempt to optimize the use of existing infrastructure and minimize the exposure of patients to the virus. ${ }^{[7]}$ While the rationale of this approach is non-controversial, several uncertainties have been raised around the issue of restarting elective surgical practice. The optimal timing of restarting, patient selection, the use of screening tools, and the optimal screening tool are undecided, as are the issues of the geographical proximity of elective 
services and emergency services in hospitals. These issues are of particular importance as concerns of a potential second wave have been announced..$^{[8]}$ These concerns have been validated by the observations of resurgence of cases and renewed lockdown in several countries including China, Spain, and North America. Restarting elective practice ${ }^{[9,10]}$ has been the subject of several medical publications, as well as the being a subject of major social and political debate in contemporary media.

The International Consensus Group has recently issued some of the main implications which should be considered and addressed while planning a return to elective service delivery. ${ }^{[9]}$ In the present study, we aimed to investigate the effectiveness of a screening questionnaire to identify high-risk patients for COVID-19 among those undergoing elective orthopedic surgery based on experiences of a highvolume arthroplasty center. In this context, answers to the following questions were sought: (i) How many patients were tested positive for SARS-CoV-2 and were classified as high risk patients? (ii) Were there patients who were screened as low-risk and were tested positive in reverse transcriptase-polymerase chain reaction (RT-PCR) diagnostics? (iii) How many planned surgeries had to be postponed to minimize exposure and transmission of SARS-CoV-2 to other patients and staff? (iv) What was the transmission rate within the hospital?

\section{PATIENTS AND METHODS}

This single-center, retrospective study was conducted at ENDO-Klinik Hamburg, a highvolume arthroplasty center, between May $4^{\text {th }}, 2020$ and June $11^{\text {th }}, 2020$. A total of 1,021 consecutive patients (492 males, 529 females; mean age: $62.3 \pm 15.1$ years; range, 13 to 91 years) who were scheduled for elective orthopedic surgery were included. A screening questionnaire was applied to all patients. The patients admitted to hospital were also tested for

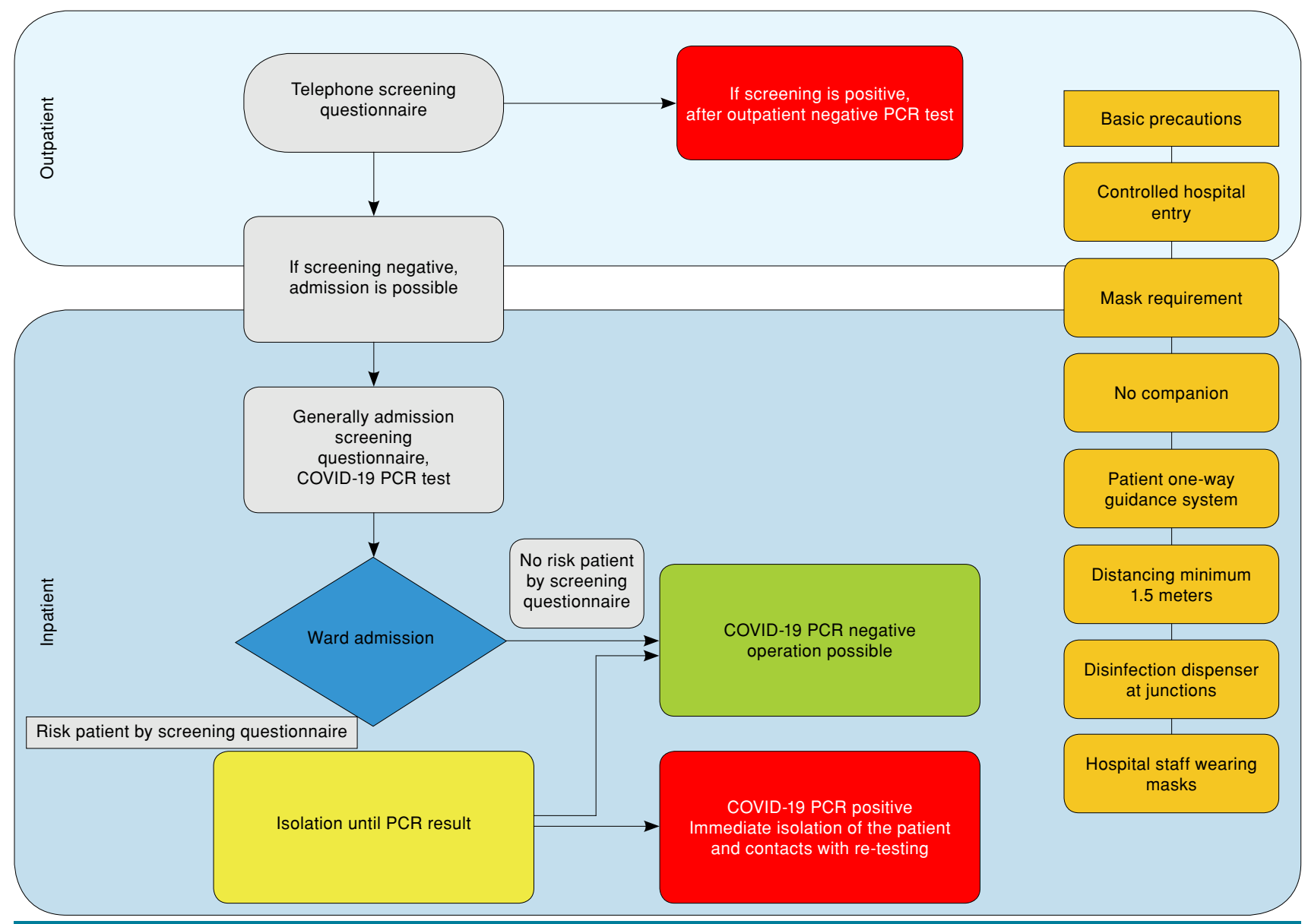


COVID-19 infection through reverse transcriptionpolymerase chain reaction of the nasopharyngeal swab and the results were documented. Demographic and clinical characteristics of the patients and operative data were recorded. A written informed consent was obtained from each patient. The study protocol was approved by the Ärztekammer Hamburg Ethics Committee (WF-112/20). The study was conducted in accordance with the principles of the Declaration of Helsinki. As described by Parvizi et al., ${ }^{[9]}$ a questionnaire for the screening of patients was implemented to resume elective surgery. Two days before the planned admission for surgery, the questionnaire was completed via telephone with the patient. This included, in addition to possible contact with COVID-19-infected individuals, symptoms such as fever, cough, pharyngitis or loss of smell (anosmia). ${ }^{[9,11-13]}$ If a question was answered positively with "Yes", the patient was classified as a patient at risk and a 14-day quarantine was implemented and a negative RT-PCR test or proof of an infection with SARS-CoV-2 was obtained later. If there were no risk factors for a positive SARS-CoV-2 infection, the patient was admitted to hospital. The questionnaire and the RT-PCR swab test were, then, re-evaluated during inpatient admission. The patients without risk factors with a negative smear were categorized into the green zone, where the patients were considered eligible for surgery. In the green zone, the basic precautions such as social distancing, wearing of a face mask, and hand hygiene are applied (Figure 1). ${ }^{[3]}$ If a patient was tested positive, the patient was immediately isolated in the red zone and their immediate contact persons (family members or neighbors) were placed in the yellow zone. In individual cases, all contact persons were tested again. The red zone represents confirmed SARS-CoV-2. In this red zone, staff wear an FFP-3 mask, hoods, protective goggles, and moisture-proof protective gowns, in addition to the basic precautions. In the yellow zone, all patients are kept who have an increased risk profile (transfer from another hospital) and have not had a confirmed negative result of the COVID RT-PCR test. Staff in the yellow zone follow the same precautions as in the red zone, except for an FFP-2 mask, instead of an FFP-3 mask.

\section{Screening questionnaire}

The SARS-CoV-2 infection is transmitted through contact with infected persons, ${ }^{[1,15]}$ most commonly presenting with symptoms such as fever, cough, fatigue, and loss of smell..$^{[9,11-13]}$ Therefore, the questionnaire includes a combination of questions about contact with potentially infected individuals and questions about the symptoms of COVID-19 disease (Table I). If at least one question was answered with "Yes", the patient was considered to be at a high risk for SARS-CoV-2 infection. If an infection was previously confirmed with a positive RT-PCR result and symptoms were absent for minimum two weeks or a negative RT-PCR result was documented after the beginning of the symptoms, the patient was classified as low risk for SARS-CoV-2 infection, despite a positive screening.

\section{SARS-CoV-2 RT-PCR testing}

Routine preoperative diagnostics included the combination of nasopharyngeal and oropharyngeal swabs for RT-PCR testing of SARS-CoV-2. ${ }^{[16,17]}$ The swab was taken with an ESwab ${ }^{\circledR}$ (COPAN Italia Spa, Via Perotti 10, Brescia, Italy) in a liquid preservation medium consisting of $1 \mathrm{~mL}$ Amies medium. The test was performed on the day of admission or the day before elective surgery.

\section{Statistical analysis}

Statistical analysis was performed using the IBM SPSS Statistics software version 27.0 (IBM Corp.,

\section{TABLE I}

SARS-CoV-2 screening questionnaire (if only one question is answered "Yes", the patient is classified in the high-risk group) SARS-CoV-2 screening questionnaire

- Have you had an infection or have you tested positive? - If "yes": Have the isolation/quarantine measures beenlifted?

Is there a negative result on SARS-CoV-2?

- Do you currently have or have you had the following symptoms in the last 14 days?

- Fever, headache, sore throat, rhinitis, dyspnea, dry cough, shivering, pain in limbs, fatigue, diarrhea, loss of smell

- Contact with people in quarantine within the last 14 days? (Applies only to people living together in the same household)

- Contact to confirmed COVID-19 case within the last 14 days?

- Has there been a stay in another hospital within the last 14 days?

- If "yes": Were there COVID-19 cases in this hospital? - If "yes": In your ward?

- Are you a resident in a nursing home, nursing flat share or mass accommodation in which there have been COVID-19 cases in the last 14 days?

SARS-COV-2: Severe acute respiratory syndrome-coronavirus 2 COVID-19: Novel coronavirus-2019. 


\begin{tabular}{|lcc|}
\hline \multicolumn{3}{|c|}{ TABLE II } \\
Overall screening results $(\mathrm{n}=1,021)$ \\
\cline { 2 - 3 } & \multicolumn{1}{|c|}{ Absolute frequencies } \\
\cline { 2 - 3 } & $\mathrm{n}$ & $\%$ \\
\hline Low-risk SARS-CoV-2 & 991 & 97 \\
High-risk SARS-CoV-2 & 30 & 3 \\
\hline SARS-COV-2: Severe acute respiratory syndrome-coronavirus 2. \\
\hline
\end{tabular}

Armonk, NY, USA). Continuous data were expressed in mean \pm standard deviation (SD) or median (min-max), while categorical data were expressed in number and frequency.

\section{RESULTS}

Of a total of 1,021 patients, 1,003 (98.2\%) underwent elective surgery as planned. During the study period, 30 of 1,021 patients answered the questionnaire with at least one question "Yes". Consequently, $30(3 \%)$ patients were classified as high risk and $991(97 \%)$ patients as low risk for a SARS-CoV-2 infection (Table II). Overall, the high-risk patients answered 49 questions with "Yes" (Figure 2).

In the high-risk group, 10 patients had a negative RT-PCR test for SARS-CoV-2 after the onset of symptoms. Another two patients were already tested positive for SARS-CoV-2 and completed the 14-day quarantine. In a total of 18 patients, surgery had to be postponed (Table III). Twelve patients with a current negative test or who already experienced SARS-CoV-2 infection were admitted for hospitalization. These 18 patients in whom surgery was postponed suffered from the listed symptoms. The most common symptom was rhinitis $(n=4)$, followed by sore throat, dyspnea, dry cough, loss of smell $(\mathrm{n}=2)$ and diarrhea, fatigue, headache and fever, all of which were reported once (Figure 2).

Of a total of 991 patients classified as low-risk and subsequently operated in our department, none of them were tested positive for SARS-CoV-2 in the RT-PCR. In addition, 12 patients with an initial highrisk classification, all were tested negative in the RT-PCR. Therefore, all 1,003 patients admitted to the inpatient admission could be operated, as scheduled.

The overall mean time of surgery was $73 \pm 15.1 \mathrm{~min}$. The mean length of hospitalization was $5.5 \pm 5.3$ days. The most frequent operations were primary total hip arthroplasty $(27 \%)$ and total knee arthroplasty (22\%) (Table IV). A revision surgery during the hospital stay was required in five patients $(0.5 \%)$. The reasons for revisions surgery were postoperative wound infection $(n=3)$, dislocation $(n=1)$, and hematoma $(n=1)$. No medical complications related to COVID-19 infection was observed in any of the patients.

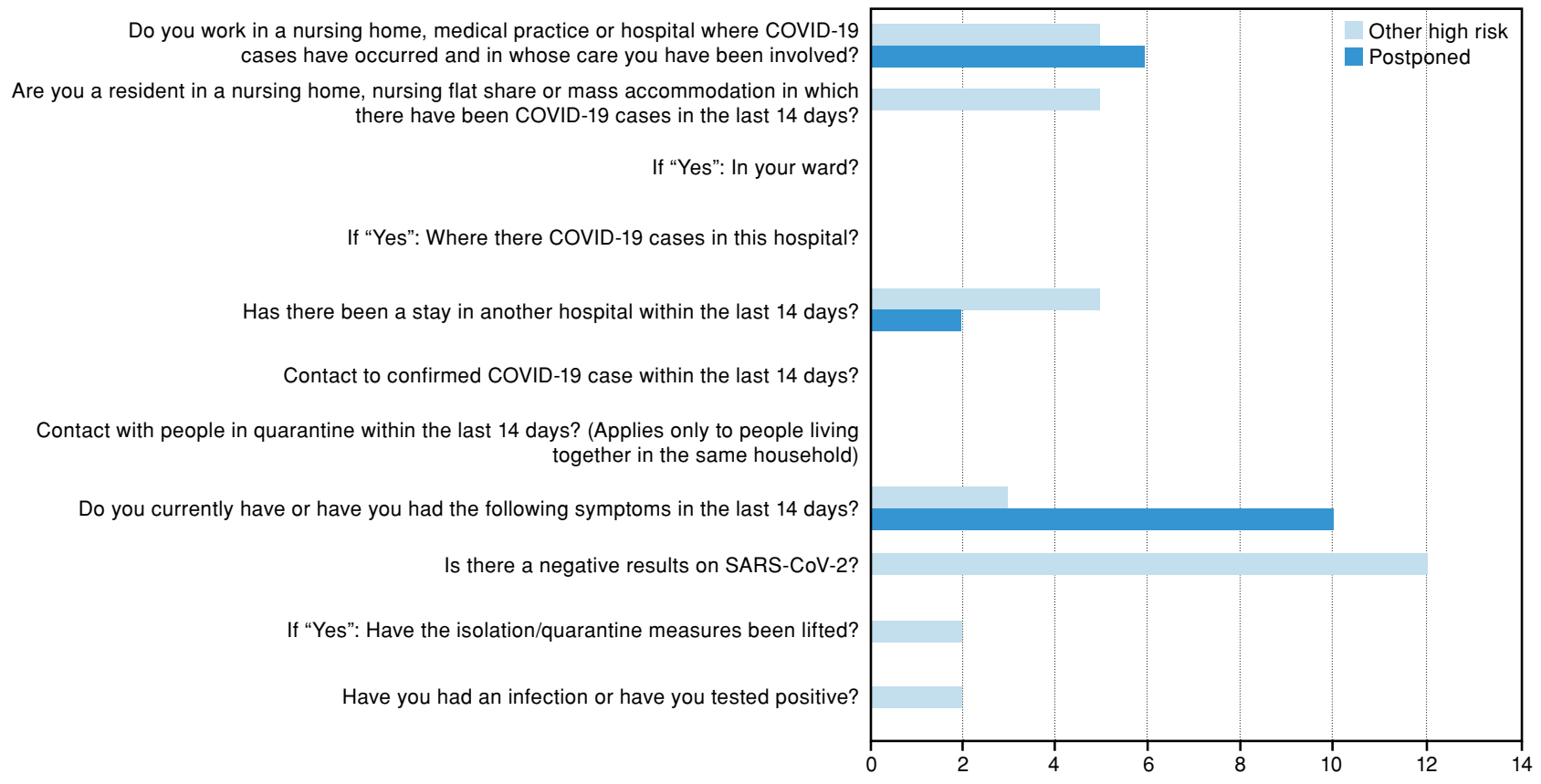




\begin{tabular}{|lcc|}
\hline \multicolumn{3}{|c|}{ TABLE III } \\
Reporting of screening results in the high-risk group $(\mathrm{n}=30)$
\end{tabular}

\section{DISCUSSION}

During the COVID-19 outbreak, there are several studies reporting recommendations for resuming elective orthopedic surgery. ${ }^{[9,10]}$ However, there is a paucity of studies examining clinical practice and outcomes after resumption of elective orthopedic surgery. To the best of our knowledge, this is the first study to report the clinical results on more than 1,000 procedures after resuming elective orthopedic surgery. Using the described interventions performed at our institution, we were able to identify low-risk patients for COVID-19 prior admission. All low-risk patients were tested negative for COVID-19. Using our hospitals implementations for resuming elective surgery, we were able to perform elective orthopedic surgeries without an increase in the postoperative complication or revision rates. All patients were discharged, as planned. At the time of discharge, all patients were COVID-19-free.
In a study, Hernigou et al. ${ }^{[18]}$ presented the first elective cases performed between May $18^{\text {th }}$, 2020 and June $14^{\text {th }}, 2020$ in Belgium. The authors concluded that resuming of elective surgical procedures seemed to be more difficult for patients than for surgeons. Only $12 \%$ of the cancelled patients during the outbreak accepted to proceed with rescheduling immediately. In our patient cohort, all patients $(n=18)$ in whom surgery was postponed could be rescheduled expediently. In the study of Hernigou et al., ${ }^{[18]}$ screening prior elective surgery was focused. The authors performed a universal testing including COVID-19 RT-PCR and thoracic computed tomography (CT) scan. Six of 211 asymptomatic patients had positive RT-PCR results, while no abnormalities were seen on thoracic CT scans.

In another study, Gruskay et al. ${ }^{[19]}$ concluded that a positive radiographic chest finding as a screening criterion did not improve the negative predictive value of screening. However, in this study, a high rate of COVID-19 infections was present in a protocol for universal testing of all orthopedic surgical admissions using nasopharyngeal swab testing. The study was carried out during the period of April $5^{\text {th }}, 2020$ to April $24^{\text {th }}, 2020$. The rate of new COVID-19 infections in the United States was high with a total of 34,272 new cases per day on April $5^{\text {th }}$ and 17,588 new cases per day on April $23^{\text {rd }}$, indicating an absolute change of $-16,684(-49 \%)$ cases by 842,629 cumulative confirmed COVID-19 cases across the country. ${ }^{[20]}$

TABLE IV

Mean surgery time and length of hospital stay according to type of surgery

\begin{tabular}{|c|c|c|c|c|}
\hline \multirow[b]{2}{*}{ Type of surgery } & \multicolumn{2}{|c|}{ Frequencies } & \multirow{2}{*}{$\begin{array}{c}\text { Mean hospital stay in days } \\
\text { Mean } \pm S D\end{array}$} & \multirow{2}{*}{$\frac{\text { Mean surgery time in minutes }}{\text { Mean } \pm S D}$} \\
\hline & $\mathrm{n}$ & $\%$ & & \\
\hline Primary THA & 271 & 27 & $5.4 \pm 1.3$ & $59 \pm 0.012$ \\
\hline Primary TKA & 219 & 22 & $5.5 \pm 1.5$ & $74 \pm 0.014$ \\
\hline Revision THA & 71 & 7 & $6.6 \pm 8.9$ & $120 \pm 0.048$ \\
\hline Revision TKA & 46 & 4.6 & $8.1 \pm 11.8$ & $162 \pm 0.049$ \\
\hline Primary TSA & 24 & 2.4 & $4.5 \pm 1.4$ & $70 \pm 0.014$ \\
\hline Revision TSA & 3 & 0.2 & $6.3 \pm 1.5$ & $126 \pm 0.036$ \\
\hline $\begin{array}{l}\text { Sports orthopaedics } \\
\text { (arthroscopy knee, shoulder etc.) }\end{array}$ & 198 & 19.8 & $2 \pm 2.7$ & $43 \pm 0.019$ \\
\hline Spine surgery & 51 & 5 & $7 \pm 5.6$ & $157 \pm 0.058$ \\
\hline Others (foot/ankle surgery etc.) & 120 & 12 & $4 \pm 5.6$ & $54 \pm 0.025$ \\
\hline Overall & 1003 & 100 & $5.5 \pm 5.3$ & $73 \pm 15.1$ \\
\hline
\end{tabular}


Interestingly, no patient in our cohort was tested positive for COVID-19 during the study period. Furthermore, all patients included in the study showed no COVID-19-associated symptoms during the hospital stay. Re-testing of the patients before discharge was not a routine procedure, but was only performed on request by the rehabilitation facilities. All retested patients were tested negative once again. However, it must be noted that the rate of new infections at the time of this study was lower in Germany with 679 new infections per day on May $4^{\text {th }}$ to 555 new infections per day on June $11^{\text {th }}$, indicating an absolute change of $-124(-18 \%)$ cases by 198 and there were a total of 178 cumulative confirmed COVID-19 cases in Germany. ${ }^{[21]}$

In our study, no medical complications related to COVID-19 following elective orthopedic procedures were encountered. Similar findings were also reported in previous studies. ${ }^{[18,19]}$ The implementation of specific measures and preoperative identifying of high-risk patients for COVID-19 and universal testing of all patients is highly recommended to avoid any catastrophic postoperative course. It has been already shown that postoperative pulmonary complications occur in half of patients with perioperative COVID19 infection. ${ }^{[22]}$ Another important issue is the preoperative selection of an adequate number of patients. In a recent study by Williamson et al., ${ }^{[23]}$ higher mortality rates related to COVID-19-infections were reported in older patients, male patients, AfroAmerican and South Asian patients, as well as in those with diabetes or severe asthma. As described by Parvizi et al., ${ }^{[9]}$ elective surgery should be riskstratified and possibly deferred on the basis of patients' comorbidities. On the other hand, it is of utmost importance to resume elective surgery during the pandemic in selected cases. ${ }^{[2]}$ According to the study by Jain et al., ${ }^{[25]}$ there would be a cumulative backlog of $>1$ million surgical cases two years after the end of elective surgery deferral.

Nonetheless, there are some limitations to this study. First, at the time of the study, the prevalence of COVID-19 infection was relatively lower in Germany. Second, we did not perform routinely a repeated RT-PCR test during the hospital stay or prior discharge. Therefore, possible positive COVID-19 cases may have not been detected during the hospital stay. Third, in our hospital, we did not perform routinely a thoracic CT scan. Asymptomatic patients or negative-tested patients could have CT abnormalities specific for COVID-19. However, the study by Hernigou et al. ${ }^{[18]}$ showed no abnormalities in asymptomatic patients. Fourth, follow-up examination of the patients was unable to be performed after discharge. It is possible that the patients may have developed symptoms or complications related to COVID-19 afterwards, since late symptom-onset may be seen. Therefore, further studies with longer follow-up are required to overcome those shortcomings. Nevertheless, the major strength of our study is the largest patient population after resuming elective orthopedic surgery in the current literature.

In conclusion, our study results suggest that a basic questionnaire in combination with a universal testing with nasopharyngeal RT-PCR is a safe screening protocol to resume elective orthopedic surgery during pandemic. The use of the guiding principles for resuming elective orthopedic surgery is safe without a higher risk for complications in selected cases. We highly recommend contacting patients prior admission using a basic questionnaire to identify the high-risk group. This patient group should be postponed or tested prior admission for COVID-19 to avoid any transmission in the hospital. All patients admitted should undergo a nasopharyngeal RT-PCR screening test for SARS-CoV-2.

\section{Declaration of conflicting interests}

The authors declared no conflicts of interest with respect to the authorship and/or publication of this article.

\section{Funding}

The authors received no financial support for the research and/or authorship of this article.

\section{REFERENCES}

1. Clark A, Jit M, Warren-Gash C, Guthrie B, Wang HHX, Mercer SW, et al. Global, regional, and national estimates of the population at increased risk of severe COVID-19 due to underlying health conditions in 2020: a modelling study. Lancet Glob Health 2020;8:e1003-e1017.

2. Gates B. Responding to Covid-19 - A Once-in-a-Century Pandemic? N Engl J Med 2020;382:1677-9.

3. Massey PA, McClary K, Zhang AS, Savoie FH, Barton RS. Orthopaedic Surgical Selection and Inpatient Paradigms During the Coronavirus (COVID-19) Pandemic. J Am Acad Orthop Surg 2020;28:436-50.

4. Health AGDo. Interim recommendations for the use of personal protective equipment (PPE) during hospital care of people with Coronavirus Disease 2019 (COVID-19). 2020. Available at: https://www.health.gov.au/sites/default/ files/documents/2020/02/interim-recommendations-forthe-use-of-personal-protective-equipment-ppe-duringhospital-care-of-people-with-coronavirus-disease-2019covid-19.pdf [Accessed: March 05, 2020]

5. Lau H, Khosrawipour V, Kocbach P, Mikolajczyk A, Schubert J, Bania J, et al. The positive impact of lockdown in Wuhan on containing the COVID-19 outbreak in China. J Travel Med 2020;27:taaa037. 
6. Phua J, Weng L, Ling L, Egi M, Lim CM, Divatia JV, et al. Intensive care management of coronavirus disease 2019 (COVID-19): challenges and recommendations. Lancet Respir Med 2020;8:506-17.

7. Pan A, Liu L, Wang C, Guo H, Hao X, Wang Q, et al. Association of Public Health Interventions With the Epidemiology of the COVID-19 Outbreak in Wuhan, China. JAMA 2020;323:1915-23.

8. Xu S, Li Y. Beware of the second wave of COVID-19. Lancet 2020;395:1321-2.

9. Parvizi J, Gehrke T, Krueger CA, Chisari E, Citak M, Van Onsem S, et al. Resuming Elective Orthopaedic Surgery During the COVID-19 Pandemic: Guidelines Developed by the International Consensus Group (ICM). J Bone Joint Surg Am 2020;102:1205-12.

10. Vannabouathong C, Devji T, Ekhtiari S, Chang Y, Phillips SA, Zhu M, et al. Novel Coronavirus COVID-19: Current Evidence and Evolving Strategies. J Bone Joint Surg Am 2020;102:734-44.

11. Giacomelli A, Pezzati L, Conti F, Bernacchia D, Siano M, Oreni $\mathrm{L}$, et al. Self-reported olfactory and taste disorders in patients with severe acute respiratory coronavirus 2 infection: A Cross-sectional study. Clin Infect Dis 2020;71:889-90.

12. Huang C, Wang Y, Li X, Ren L, Zhao J, Hu Y, et al . Clinical features of patients infected with 2019 novel coronavirus in Wuhan, China. Lancet 2020;395:497-506.

13. Chen N, Zhou M, Dong X, Qu J, Gong F, Han Y, et al. Epidemiological and clinical characteristics of 99 cases of 2019 novel coronavirus pneumonia in Wuhan, China: a descriptive study. Lancet 2020;395:507-13.

14. Ye G, Pan Z, Pan Y, Deng Q, Chen L, Li J, et al. Clinical characteristics of severe acute respiratory syndrome coronavirus 2 reactivation. J Infect 2020;80:e14-e17.

15. Kannan S, Shaik Syed Ali P, Sheeza A, Hemalatha K. COVID-19 (Novel Coronavirus 2019) - recent trends. Eur Rev Med Pharmacol Sci 2020;24:2006-11.

16. (CDC) CoDCaP. Testing for COVID-19. [updated Updated June 24, 2020]; Available at: https://www.cdc.gov/ coronavirus/2019-ncov/symptoms-testing/testing.html.
17. Kim D, Quinn J, Pinsky B, Shah NH, Brown I. Rates of Co-infection Between SARS-CoV-2 and Other Respiratory Pathogens. JAMA 2020;323:2085-6.

18. Hernigou J, Valcarenghi J, Safar A, Ferchichi MA, Chahidi E, Jennart $\mathrm{H}$, et al. Post-COVID-19 return to elective orthopaedic surgery-is rescheduling just a reboot process? Which timing for tests? Is chest CT scan still useful? Safety of the first hundred elective cases? How to explain the "new normality health organization" to patients? Int Orthop 2020;44:1905-13.

19. Gruskay JA, Dvorzhinskiy A, Konnaris MA, LeBrun DG, Ghahramani GC, Premkumar A, et al. Universal Testing for COVID-19 in Essential Orthopaedic Surgery Reveals a High Percentage of Asymptomatic Infections. J Bone Joint Surg Am 2020;102:1379-88.

20. (ECDC) ECfDPaC. The number of confirmed cases is lower than the number of actual cases; the main reason for that is limited testing.: European Centre for Disease Prevention and Control (ECDC); 2020 [updated 25.07.2020; cited 2020 25.07.2020]; Available at: https:// github.com/owid/covid-19-data/tree/master/public/ data.

21. Gralnek IM, Hassan C, Beilenhoff U, Antonelli G, Ebigbo A, Pellisè $M$, et al. ESGE and ESGENA Position Statement on gastrointestinal endoscopy and the COVID-19 pandemic. Endoscopy 2020;52:483-90.

22. COVIDSurg Collaborative. Mortality and pulmonary complications in patients undergoing surgery with perioperative SARS-CoV-2 infection: an international cohort study. Lancet 2020;396:27-38.

23. Williamson EJ, Walker AJ, Bhaskaran K, Bacon S, Bates C, Morton CE, et al. Factors associated with COVID-19-related death using OpenSAFELY. Nature 2020;584:430-6.

24. Atik OŞ. Elective surgeries during COVID-19 storm: The best surgeon knows when not to operate. Jt Dis Relat Surg 2020;31:161-2.

25. Jain A, Jain P, Aggarwal S. SARS-CoV-2 Impact on Elective Orthopaedic Surgery: Implications for Post-Pandemic Recovery. J Bone Joint Surg [Am] 2020;102:e68. 